\title{
Sensation und Sensibilität
}

Zu John Lockes Einfluß auf das Konzept der Sensibilität im 18. Jahrhundert

Von Antoinette Stettler

\section{Einleitung}

Es wird gemeinhin das 18. Jahrhundert auch als das Jahrhundert der Sensibilität, der Empfindsamkeit, charakterisiert, die dann auch in die Empfindelei einer überfeinerten Romantik geführt habe. In der Tat ist im 18. Jahrhundert in vielen Gebieten der Kultur eine gesteigerte Sensibilität zu beobachten, die sich auch in einem erhöhten Subjektivismus äußert. Nicht nur finden wir in der Philosophie die Richtung des Sensualismus, in der Literatur vermehrt die Selbstbeobachtung, auch in der Malerei drückt eine erhöhte Sensibilität für das Landschaftserlebnis eine mehr gefühlsbetonte Sicht aus u.a.m.

Sensibilität drückte in diesem Sinne eine psychische Qualität aus, so wie das Wort mithin auch heute noch gebraucht wird.

Sensibilität besitzt aber zusätzlich zum psychischen Gehalt auch noch einen körperlichen, der heutzutage beinahe nur noch in der Medizin, namentlich in der Neurologie und Physiologie verwendet wird.

Dazu zur Illustration folgendes Zitat aus dem Konversationslexikon DTV von 1980:

«Sensibilität (Physiologie): Allgemein die Fähigkeit, auf Reize nicht nur irgendwie zu reagieren (Irritabilität), sondern diese auf dem Weg über sensible Nerven und Sinneszentren der Großhirnrinde mit seelischen Vorgängen (Empfindungen, Wahrnehmungen) zu beantworten. Speziell unterscheidet man die auf dem Tastsinn beruhende Sensibilität der Haut (Oberflächensensibilität) von der auf dem kinästhetischen Sinn beruhenden Sensibilität der Gelenke, Sehnen, Muskeln (Tiefensensibilität).»

Von der psychischen Seite der Sensibilität ist da gar nicht mehr die Rede.

Es soll nun in der vorliegenden Arbeit versucht werden, den Stellenwert zu bestimmen, den der Begriff Sensibilität im 18. Jahrhundert für die Medizin innehatte. Dazu müssen wir bis ins Jahr 1690 ausholen, als John Locke's «Essay Concerning Human Understanding» erschien. 


\section{John Locke, Thomas Willis und die «tabula rasa»}

John Locke (1632-1704) ist als Philosoph berühmt geworden. Sein Hauptwerk, «An Essay Concerning Human Understanding», wurde 1690 veröffentlicht. Locke begründete darin die Philosophie des englischen Empirismus. Er bekämpfte die platonische Lehre der angebornen Ideen und ließ als Erfahrungsquelle nur die Sinnesempfindung («sensation») und die Selbstwahrnehmung («reflection») zu. Die wahrnehmbaren Sinnesqualitäten sind subjektiv, die Sprache ein System von Zeichen, welche die Vorstellungen und ihre Beziehungen vertreten. Mathematik und Ethik erreichen einen höheren Grad von Gewißheit als die Naturwissenschaften. Seine politischen Schriften, die über Montesquieu für die französische Revolution einflußreich wurden, sollen hier nicht behandelt werden.

Daß Locke in seinen jüngeren Jahren auch experimentierender und bis an sein Lebensende praktizierender Arzt war, ist weniger bekannt. Auf Anraten von Richard Lower (1631-1691) begann Locke in Oxford das Medizinstudium $^{1}$. 1652, als Locke im Christ Church College in Oxford Student wurde, war die Universität nach den Cromwellschen Bürgerkriegswirren aristotelisch/orthodox geblieben und zudem puritanisch geworden. Lockes Studium begann demnach mit den traditionellen Fächern, d.h. mit einem kaum modifizierten Galenismus.

Neben der offiziellen Medizin existierte in Oxford aber auch der «experimental philosophical club» oder das «invisible college», wie es auch genannt wurde, dem drei Freunde Lockes angehörten. Erstens war das der schon genannte Richard Lower, der sich durch anatomische, physiologische und chemische Entdeckungen auszeichnen sollte; ferner Christopher Wren (1632-1723), der anhand anatomischer Modelle die Muskelmechanik abzuklären suchte und später seinen Ruhm als Kirchenbauarchitekt von St. Paul's usw. in London gewann; schließlich Robert Hooke (1635-1703), der zunächst Thomas Willis (1621-1675) bei dessen hirnanatomischen Sektionen half und später ein hervorragender Mikroskopist wurde.

Das «invisible college» machte sich die Experimentiermethode William Harveys (1578-1657) zu eigen, der bekanntlich auf diese Weise 1628 den Blutkreislauf entdeckte. Der spiritus rector dieses Experimentierklubs war ohne Zweifel Robert Boyle (1627-1691), der u.a. die Atmungsphysiologie studierte, und dem beinahe die Entdeckung der Rolle des Sauerstoffes bei der Verbrennung gelungen wäre. Das «invisible college» tagte informell, sei es an den verschiedenen Wohnorten oder in den Laboratorien der Teilhaber 
und hatte eingerechnet der an den Experimenten Zuschauenden, die man auch «virtuosi» nannte, rund 50 Mitglieder. Aus diesen Versammlungen ging 1660 die Royal Society in London hervor.

Zu dieser Gemeinschaft stieß Locke bald nach Beginn seiner Medizinstudien. Man gewinnt den Eindruck, daß er den größeren Teil seiner Zeit in diesem Kreis verbrachte und wenig an den offiziellen Veranstaltungen der Universität teilnahm, die ihm denn auch zeitlebens nur das Bakkalaureat und nicht die Doktorwürde verlieh.

Wie aus dem Gesagten hervorgeht, waren die Interessen dieser Gelehrtengemeinschaft sehr weitgefächert, wie das kürzlich Robert G. Frank ${ }^{2}$ dargestellt hat. Von Locke, der sich doch wohl vorwiegend auf der Zuschauerseite befand, wissen wir, daß ihn speziell die Hirnanatomie interessierte. Wir besitzen von ihm Vorlesungsnotizen, in einer selbsterfundenen Stenographie, die er in den Vorlesungen des erwähnten Thomas Willis erstellte ${ }^{3}$. Auch besaß Locke des Willis «Cerebri Anatome» (1664).

Nach seiner Zeit in Oxford wurde Locke von 1667-1675 Hausarzt bei David Ashley in London, der später als Earl of Shaftesbury Lordkanzler von England wurde. Standen zunächst noch Laboratoriumsversuche, an denen sich auch Ashley beteiligte, im Vordergrund, verband sich Lockes Tätigkeit mehr und mehr mit den politischen Schicksalen seines Brotherrn. In diese Zeit fällt auch Lockes Freundschaft mit Thomas Sydenham (1624-1689), den er oftmals auf seinen Arztvisiten am Krankenbett begleitete. Gemeinhin wird angenommen, daß in dieser Ärztefreundschaft Sydenham der Gebende und Locke der Nehmende war, d.h. daß Locke vom mehr basiswissenschaftlichen Denken seiner Oxforder Freunde weg zum hippokratischen Beobachten der Krankheitsphänomene und deren (chemischer) Therapie geführt wurde $^{4}$. Beide, Sydenham und Locke, scheinen von der Mikroskopie zur Krankheitsaufklärung nicht viel gehalten zu haben ${ }^{5}$. Neuerdings wird aber auch anhand der verschiedenen, von Sydenham selbst revidierten Ausgaben seiner Epidemielehre auf einen sehr großen Einfluß Lockes hingewiesen ${ }^{6}$.

Die Jahre 1675-1679 verbrachte Locke auf einer Frankreichreise, wo er in Montpellier vor allem mit Charles Barbeyrac (1629-1699) in medizinische Verbindung trat. Barbeyrac verfolgte ein ähnliches Ziel wie Sydenham, nämlich die Medizin auf ein hippokratisches Konzept zu stellen, so daß schon vermutet worden ist, Sydenham könnte bei Barbeyrac in Montpellier gelernt haben. Das ist aber zu verneinen ${ }^{\text {? }}$ Vielmehr verhält es sich so, daß Locke bei seiner Rückkehr in Sydenham bestätigt sah, was er von Barbeyracs Methode erfahren hatte. Auch in Paris verkehrte Locke in ärztlichen 
und naturwissenschaftlichen Kreisen. Schon in Frankreich begann Locke ein medizinisches Tagebuch, das er nach seiner Rückkehr bis fast an sein Lebensende weiterführte. Politisch war er nach seiner Rückkehr weiterhin mit dem Earl of Shaftesbury verbunden, welcher den Restaurationsbestrebungen Jakobs II Stuart feindlich gegenüberstand. In der Folge mußte Locke nach seines Protektors Tod 1683 ins holländische Exil, von wo er nach Wilhelm von Oraniens englischer Thronbesteigung (1688) wieder nach England zurückkehren konnte. Er übernahm einige politische Ämter. Seinen Lebensabend verbrachte er auf dem Lande, jedoch immer noch in regem Kontakt mit Ärzten, und sei es auch der geringste Landpraktiker.

Locke hat auch vier medizinische Schriften verfaßt: De arte medica, 1669, publ. 1876; Anatomie (inkomplett) 1668; Tussis, o. J.; Respirationis usus, o. J. ${ }^{7 \text { a }}$

Daß Locke mit der Medizin nicht nur in losem Kontakt stand, scheint aus dem Gesagten klar genug hervorzugehen. Was hat das nun aber mit seiner Philosophie zu tun?

Es ist schon pauschal gesagt worden, daß Lockes Philosophie ganz von der Medizin bestimmt gewesen sei, eine Aussage, die schon deshalb nicht gelten kann, weil seine politischen und pädagogischen Schriften mit Sicherheit eine andere Quelle haben. Anders stellt sich die Lage dar, wenn von seinem «Essay Concerning Human Understanding», seiner Erkenntnistheorie, die Rede ist. Begonnen 1670, wurde das «Essay» 1690 publiziert und hat zum großen Teil Lockes Berühmtheit begründet. Er selbst nennt Boyle, Huygens, Newton und Sydenham als seine Inspiratoren. Alle vier sind Empiriker, sei es durch das Experiment wie die drei Erstgenannten, sei es durch die reine, der Spekulation abholde Beobachtung des unbeirrbaren Sydenham. Hat Locke einen weiteren seiner Inspiratoren zu erwähnen vergessen? Wir meinen den Hirnforscher Thomas Willis, dessen Vorlesung sich Locke, wie erwähnt, notiert hatte.

Willis war wohl der bedeutendste Hirnforscher des 17.Jahrhunderts. Neun Jahre älter als Locke, gehörte auch er zum «invisible college». Wir besitzen von ihm nebst «Cerebri Anatome» (1664), «De morbis convulsivis» (1667) und «De anima brutorum» (1672), eine komparative Hirnanatomie ${ }^{8}$. Nicht nur geht die Entdeckung der arteriellen Hirnzirkulation (circulus arteriosus Willisii) auf ihn zurück. Er ist auch der gedankliche Erfinder des Reflexbogens, über dessen detaillierten Verlauf ja noch heute geforscht wird. Der Reflex ist ihm Antwort auf einen Schmerzreiz. Er vermutete, daß die Basalganglien, vor allem das Corpus striatum, eine wichtige Funktion in 
der Verarbeitung der Sinnesempfindungen habe, ja er nahm sogar an, daß das Corpus striatum der Sitz des «sensorium commune» sei. Die Reizleitung im Nerv dachte sich Willis lichtschnell, die Reaktion im Muskel als Explosion iatrochemischer Natur. Der galenische «spiritus animalis» war für den Atomisten Willis ein feinstmaterielles Agens, das nur aus der Funktion zu erkennen sei $^{9}$.

Interessant ist nun, was die eingangs erwähnten Vorlesungsnotizen Lockes über die Vorstellungen von der Funktion des Nervensystemes enthalten. Locke schreibt, vermutlich 1663 oder 1664, folgendes:

\footnotetext{
"Senses: Imagination is caused by an impression of some external object that moves the spirits inwards and excites other spirits in the medulla oblongata into an expansive movement. These latter spirits then are variously circulated through the cerebral orbit forming different ideas. When a similar movement is repeated in these orbits memory is evoked, as the cerebrum in infants is a tabula rasa" (im Original gesperrt) ${ }^{10}$.
}

Wenden wir uns nun dem «Essay» Lockes zu, das auf philosophischer Basis versucht, den Erkenntnisvorgang zu durchleuchten. Im ersten Buch begründet er, weshalb die platonische Vorstellung von den angeborenen Ideen nicht stimmen könne. Im zweiten Buch kommt sodann die Rede auf Lockes eigene Vorstellung vom Erkenntnisvorgang. Dort steht folgendes:

\footnotetext{
"Let us then suppose the mind to be, as we say, white paper, void of all characters, without any idea; how come it to be furnished? Whence comes it by that vast store which the busy and boundless fancy of man has painted on it with an almost endless variety? Whence has it all the materials of reason and knowledge? To this $I$ answer in one word, from experience; in that all our knowledge is founded and from that it ultimately derives itself." 11
}

und

"This great source of most of the ideas we have, depending wholly upon our senses, and derived by them to the understanding, I call sensation". ${ }^{12}$

Ausgehend vom Geist als weißem Blatt Papier und der Sinnesempfindung baut Locke seine sehr komplexen Vorstellungen auf, wie es zur Wahrnehmung, Reflexion, Gedächtnis, Wille usw. kommt. Darauf müssen wir hier nicht eingehen. Beizufügen bleibt, daß Locke sich nirgends über die Kräfte, die spiritūs, äußert, die alle diese Vorgänge in Betrieb setzen.

Uns kommt es hier vielmehr darauf an, auf die frappante Ähnlichkeit der Gedankenführung in Lockes Vorlesungsnotizen und in seinem «Essay» hinzuweisen. Beide Male ist der Geist bei der Geburt mit dem Bild völliger Leere charakterisiert; bei Willis ausgedrückt mit der antiken Wachsschreibtafel, von der die eingeritzten Zeichen ausgelöscht worden sind; bei Locke 
mit dem zeitgenössischen weißen Papierbogen, den es zu beschreiben gilt. Beide halten die Sinnesorgane für die Ursache der Füllung des Geistes; Willis vielleicht für die Füllung der Gehirnstrukturen, Locke für den Geist schlechthin.

Darf man nun den Hirnanatomen Willis für den Inspirator Lockes in bezug auf seine Erkenntnistheorie halten? Zur Beantwortung dieser Frage ist zunächst kurz auf den Begriff der «tabula rasa» einzugehen. Dieser Ausdruck bedeutete schon für die Stoiker die geistige Leere, die mit der Sinnesempfindung gefüllt werden mußte. Auch für die Scholastiker gab es die «tabula rasa». Doch ist in diesen beiden philosophischen Systemen der Begriff eher beiläufig erwähnt und hat keineswegs den Stellenwert, den er in Lockes «Essay» bekommen sollte. Für Locke ist die «tabula rasa» recht eigentlich der Punkt, von dem sein Philosophieren den Ausgang nimmt. Er hörte von der «tabula rasa» in Willis Vorlesung.

Wie kommt nun aber Willis dazu, vom Gehirn der Neugeborenen als der «tabula rasa» zu reden? Mit dem besseren Bekanntwerden der Anatomie des Nervensystems, insbesondere der Sinnesorgane, gewann die Auseinandersetzung zwischen Platons Ideenlehre und der ebenfalls vorhandenen Lehre der «tabula rasa» eine neue, erhöhte Aktualität. Das ebenfalls erwachte allgemeine Interesse an der Empirie wirkte in die gleiche Richtung. Man darf sagen, daß zu Beginn des 17. Jahrhunderts der Begriff der «tabula rasa» zu einer eher geläufigen Argumentationsbasis wurde, ja daß sie zu Willis Zeit zum Allgemeinplatz geworden war. Das wird aus den Werken schon von Erasmus, Descartes, van Helmont, Gassendi und auch von Hobbes ersichtlich. Das «invisible college» in Oxford hat dabei keine Ausnahme gemacht.

Wenn nun also Locke in seinem «Essay» gar nicht auf die anatomischen Kenntnisse eingeht, die er sich bei Willis erworben hatte, so tat er das, meinen wir, weil für ihn des Willis Ansicht von der «tabula rasa» so selbstverständlich war, daß er sie eben dieser Selbstverständlichkeit wegen zum Ausgangspunkt seines «Essays» nahm. Lockes in der Jugend gewonnenes anatomisches Wissen floß demnach ohne weitere Hinterfragung in sein späteres philosophisches Werk als Axiom ein. Insofern kann der eingangs erwähnten Vermutung zugestimmt werden, daß Locke ein von der Medizin in ihrer anatomischen Ausprägung beeinflußter Philosoph gewesen sei.

Wie groß die Wirkung Lockes auf die Philosophen des 18. Jahrhunderts war, sei vermerkt. Wir nennen Berkeley und Hume sowie die französischen Sensualisten im Kreise um die «Encyclopédie». Aber nicht nur die Philosophen bauten auf Locke auf. Vielmehr fand sein Konzept der «sensation», 
welche das weiße Papier des Geistes erst füllt, auch in ärztlichen Kreisen großes Echo. Wie sonst wäre die Vorliebe der Physiologen des 18. Jahrhunderts, allen voran Haller, für Fragen der Sensibilität zu verstehen? Wie sonst wäre es zu Verallgemeinerungen der Wichtigkeit des Nervensystems gekommen, gipfelnd in der Neurosenlehre eines Cullen? Noch nach der französischen Revolution bezieht sich der Arzt Cabanis ausdrücklich auf Locke, als er seine Abhandlung über die «Rapports du physique et du moral de l'homme» (1802) schrieb. Was Ausgang nahm in Willis Vorlesung über Gehirnanatomie wurde durch Lockes «Essay» wiederum fruchtbar für die Ärzteschaft eines ganzen Jahrhunderts.

\section{Diskurs über die Sensibilität im 18. Jahrhundert}

Als John Locke 1690 mit seinem «Essay» an die Öffentlichkeit trat, in welchen er die «tabula rasa» und die «sensation», den Reiz oder Stimulus der Sinnesorgane, zum Urprinzip allen Verstehens und Denkens erklärte, erregte er in der gelehrten Welt großes Aufsehen.

Sein Gedankengut fand in England, wie erwähnt, in den Philosophen Berkeley und Hume seine Nachfolger. In Deutschland hat sich Leibniz damit auseinandergesetzt. Nach Frankreich kam es vor allem durch die Vermittlung von Voltaire.

In unserem Zusammenhang interessiert uns die Rezeption Lockes in Frankreich, weil dort mit den Enzyklopädisten und Jean-Jacques Rousseau der Diskurs um die Sensibilität im weitesten Sinne neue Dimensionen gewann.

Es sei vorweggenommen, daß sich der Begriff Lockes von der «sensation» nicht mit dem von z. B. Rousseaus «sensibilité» deckt. Aber beide sind voneinander abhängig. Während «sensation» etwas Spezielles meint, nämlich eben den Reiz, den die Außenwelt auf unsere Sinnesorgane ausübt, stellt «sensibilité» etwas mehr Generelles dar, gewissermaßen die Abstraktion oder Zusammenfassung aller «sensations».

Seit dem Beginn des 18. bis in die Mitte des 19. Jahrhunderts können wir bei Ärzten und Naturkundigen eine meist schriftlich geführte Diskussion um die beiden Begriffe feststellen, der wir anhand einiger Beispiele nachgehen wollen. 


\subsection{Sensation und Sensibilität in der Philosophie}

Wir erwähnen zunächst den Philosophen Etienne Bonnot de Condillac und wenden uns seinem «Traité des sensations» von $1754 \mathrm{zu}$. Condillac löste damit eine eigentliche Bewegung zur Beobachtung der Sinnesempfindung aus. Sein «Traité» behandelt - wir würden heute sagen - ein Psychogramm der menschlichen Seele, ausgehend von den fünf Sinnesempfindungen. Er braucht das Bild einer Marmorstatue, die sich unter Zufügung je eines Sinnesorganes belebt. Zuerst kommen der Tast- und der Geruchsinn; es folgen dann Gehör- und Gesichtssinn. Ganz überwiegender Anteil an der Erfassung der Außenwelt kommt dem Tastsinn zu. Er allein kann über die Gegenstände Aufschluß geben. Als Anhängsel der Tastempfindung tritt der Schmerz hinzu. Ideen, was das gleiche ist wie Erinnerungen, werden durch komplexe Ordnung der Sinnesempfindungen geformt.

«Une sensation n'est point encore une idée, tant qu'on la considère comme un sentiment, qui se borne à modifier l'âme. Si j'éprouve actuellement une douleur, je dis, que je la sens.») ${ }^{13}$

Zusammen mit Julien Offroy de La Mettrie, der seine sensualistisch/materialistischen Werke, z. B. «L'homme machine» (1748), schrieb und darin eine Ansicht vertrat, welche die menschlich/mechanische Maschine durch die Sinnesorgane angetrieben sah, wurde Condillac zu einem der Hauptbegründer des französischen Sensualismus. Dieser wirkte wiederum stark auf die Enzyklopädisten um Diderot. Condillacs und La Mettries Gedankengänge fußen weitgehend auf Lockes Philosophie, den Condillac auch immer wieder erwähnt.

Charles Bonnet (1720-1793), der berühmte Genfer Naturforscher, kam unabhängig von Condillac auf das Bild der zu belebenden Marmorstatue ${ }^{14}$. Die Wichtigkeit der Sinnesorgane und damit der Sinnesempfindung zur Belebung der geistigen und seelischen Funktionen muß demnach im 18. Jahrhundert ein Topos gewesen sein. Dabei sei angemerkt, daß bei der Sensation hauptsächlich vom Tastsinn gesprochen wurde, man aber damals noch weit entfernt war, die anatomischen Strukturen der Tastorgane zu kennen.

\subsection{Sensibilität im Experiment}

Albrecht von Haller, der Frage der Sensibilität praktisch nachgehend, machte mit der «sensation» Lockes Ernst. In unzähligen Tierversuchen 
trachtete er herauszufinden, welche Organe denn nun wirklich Empfindung besitzen. In seinem Traktat «De partibus sensilibus et irritabilibus corporis humani» (1752) gab er eine Einteilung der Gewebe in solche, die sensibel sind, d.h. einen Tastsinn haben, und in solche, die keinen besitzen. Kritisch muß zu Hallers Versuchen bemerkt werden, daß er an den Tieren mit gröbsten Schmerzreizen, wie schneiden, stechen, brennen, mit Säure behandeln usw., experimentierte. Aber da der Schmerz als Anhängsel des Tastsinnes galt, schloß er vom Schmerzreiz auf den Tastsinn zurück. Das war, wie sich im 20. Jahrhundert zeigen sollte, ein Kurzschluß, da wir heute wissen, daß Schmerz und Getast zwei verschiedene Sinnesqualitäten sind. Daß der Tastsinn mit der damals anatomisch identifizierten Nervenstruktur einen Zusammenhang hatte, war für Haller, wie schon sein Lehrer Hermann Boerhave in Leiden postuliert hatte, dabei außer Zweifel.

«Empfindlich nenne ich einen solchen Teil des menschlichen Körpers, dessen Berührung sich die Seele vorstellet ... Bei den Tieren ist es der Schmerz, von deren Seele wir nicht soviel erkennen können», und «Unempfindlich nenne ich hingegen jene Teile, bei welchen, wenn sie gleich gebrannt, gehauen, gestochen und bis zur Zerstörung geschnitten werden, kein krampfhaftes Zucken und keine Veränderung in der ganzen Lage des Körpers erreget wird .... ${ }^{15}$

Hallers Tierversuche machten großes Aufsehen, wurden nachgeahmt und blieben keineswegs unbestritten ${ }^{16}$. Man möchte meinen, daß die Diskussion um die Sensibilität durch Haller in eine handfeste Richtung geleitet wurden. Aber nichts dergleichen war der Fall; vorab entstand ein Gelehrtenstreit. Einzig, daß der Tast- und Schmerzsinn mit dem Nervensystem eng verbunden waren, blieb allgemein anerkannte Erkenntnis.

\subsection{Sensibilität als Allgemeinwissen}

\subsubsection{Henri Fouquet und der Vitalismus}

Schauen wir nun Haller gegenüber an, was die Enzyklopädisten zur Sensibilität zu sagen hatten. Die «Encyclopédie», wir rufen es in Erinnerung, heißt mit vollem Titel «Encyclopédie ou Dictionnaire raisonné des sciences, des arts et des métiers par une société des gens de lettres». Sie erschien seit 1751 und war 1778 mit 28 Foliobänden fertig, wovon 17 Bände Text und 11 Bände Abbildungen. Seit 1777 kamen 5 Supplementbände dazu. In Genf, Lausanne, Bern und Yverdon wurden weitere Auflagen dieses Riesenwerkes, das für das lernbegierige Publikum geschrieben wurde, publiziert. Die «gens de lettres» waren neben Diderot, Voltaire, d'Alembert, 
Buffon, Montesquieu, Condillac, Mably, Duclos, Turgot, Hélvetius und viele andere mehr.

Für den Artikel «sensibilité» zeichnete Henri Fouquet (1727-1806). Er war Professor in Montpellier, ein Schüler und Freund des berühmten Théophile de Bordeu, der seinerseits von François Boissier de Sauvages die Stahlsche Doktrin des Vitalismus übernahm und an der Universität von Montpellier dozierte ${ }^{17}$.

Bevor nun auf die Lehre der Sensibilität, wie sie Fouquet in der Enzyklopädie darlegt, eingegangen werden soll, ist ein kurzer Exkurs auf den Vitalismus Stahlscher Prägung angezeigt, so wie er in Montpellier gelehrt wurde. Als Reaktion gegen die Iatromechaniker, -physiker und -mathematiker, die vor allem in England, Italien und der Schule Boerhaves in Leiden wirkten, entwickelte Georg Ernst Stahl, Halle 1707, seine «Theoria medica vera», in welcher er für das Agieren der lebendigen Welt eine eigene Kraft anzunehmen genötigt war, eben die Vitalkraft. Insofern bleibt er der antiken Spirituslehre treu; nur wollte er statt der drei antiken «spiritus» nur noch eine gelten lassen. Alle lebendigen Phänomene sind auf die Vitalkraft zurückzuführen, wobei der «anima», der Seele, die leitende Funktion zugehört. Stahls Spiritus/Anima-Lehre hatte in Deutschland großen Erfolg, in England und Paris so gut wie gar keinen. Der schon erwähnte Haller kannte Stahls Lehre und bekämpfte sie aufs entschiedenste.

Nicht so die Medizinschule von Montpellier. Dort entwickelte sich eine Kontroverse zwischen Vitalisten - wie man die Stahl-Anhänger nannte und Antivitalisten, die sich von den Disputationen der Professoren bis zu studentischen Kämpfen hinzog ${ }^{17}$. In Montpellier war die vitalistische Doktrin folgendermaßen zu fassen: Man vertrat, gemessen mit Stahl, einen gemäßigteren Vitalismus und ließ auch mechanistische Anschauungen gelten. So steht z. B. Théophile de Bordeus Werk «Recherches anatomiques sur les glandes» (1752) zwischen mechanistischer und vitalistischer Auffassung. Sein dermaliger Nachfolger, Paul Joseph Barthez (1734-1806), war wiederum mehr vitalistisch eingestellt.

Wenn wir nun auf Henri Fouquet und seinen Artikel über die «sensibilité» in der Enzyklopädie zurückkommen, so ist zunächst festzustellen, daß sie über zwanzig Seiten Folioformat aufweist, also höchst ausführlich ist. Es soll nun eine kurze Zusammenfassung des Artikels folgen. Es ist darin die Rede vom Unterschied der Sensibilität und Motilität; von den Ursachen der Sensibilität auf pathologische Erscheinungen der verschiedensten Art, d.h. eine ganze Pathologie, die auf der Sensibilität aufgebaut ist; vom Einfluß der 
Klimata auf die Sensibilität; vom Unterschied der Geschlechter hinsichtlich der Sensibilität; von der Abhängigkeit der Lebensalter auf die Sensibilität: kurz, die Sensibilität vermag beinahe alle menschlichen Erscheinungen zu erklären.

«Sensibilité, sentiment: (Médecine), la faculté de sentir, le principe sensitif ou le sentiment même des parties, la base et l'agent conservateur de la vie, l'animalité par excellence, le plus beau, le plus singulier phénomène de la vie...» ${ }^{18}$

«La base et l'agent conservateur de la vie» ist nun ein weiter Begriff, der sich dem der Vitalkraft deutlich nähert. Das Interessante ist aber, daß sich Fouquet keineswegs auf den in Montpellier dozierten Vitalismus bezieht, sondern immer wieder Locke als Kronzeugen beruft. Für Fouquet war demnach die Locksche «sensation» und nicht die Stahlsche Vitalkraft der Kardinalbegriff. Er stellt sich damit auf den Boden der englischen Empirie, obwohl sich im Einzelnen seiner Argumentation noch viel Traditionelles befindet. Auch für Fouquet ist der Schmerz Teil des Tastsinnes; und auch für ihn verbindet sich die Sensibilität mit den immer besser bekannt werdenden Nervenstrukturen.

\subsubsection{Albrecht von Haller}

In der Quarto-Ausgabe der Enzyklopädie, die seit 1774 in Yverdon herausgegeben wurde, befindet sich ebenfalls ein Artikel mit dem Titel «sensibilité, sentiment». Er ist in dieser Ausgabe nicht von Henri Fouquet geschrieben, sondern mit HdG gezeichnet, was allgemein als Haller de Goumoens interpretiert wird. Goumoens ist ein kleiner Landsitz im Waadtland, den sich Haller nach seiner Rückkehr aus Göttingen erwarb. Es ist also der große Haller, der nach seinen unzähligen Tierversuchen über die Sensibilität zu Worte kommt. Man weiß aus einem Brief von Haller an Samuel Gessner in Zürich, daß Haller den Auftrag bekommen hatte, «pour ces messieurs de l'encyclopédie» einen Artikel über die Sensibilität zu schreiben ${ }^{19}$. Es war nach 1752, nach seiner Veröffentlichung der Tierversuche.

Wenn wir den Artikel untersuchen, entspricht er nicht den Erwartungen. Denn zu ungefähr $80 \%$ übernimmt er den Text von Henri Fouquet wortwörtlich. In einem letzten Teil kommt dann Haller allerdings auf seine eigenen Versuche zu sprechen, die er zwar nicht sui generis, sondern als Kontroverse mit seinen Gegnern darstellt. Von sich redet Haller in der dritten Person und fügt gerne das Epitheton ornans «cet illustre savant» hinzu. 
Die Sensibilität bleibt demnach auch für Haller «la base et l'agent conservateur de la vie», so wie er den Begriff von Fouquet übernahm. Für diesen Artikel Hallers zeigt sich ebenfalls das typische Gemisch zwischen den Begriffen der Sensibilität, Vitalkraft und dem des Schmerzes.

In den Supplementbänden zur ersten Auflage der Enzyklopädie in Folio erscheint nach 1777, dem Todesjahr Hallers, wiederum ein Artikel «sensibilité», diesmal ohne den Zusatztitel «sentiment», aber dafür in Klammer «physiologie». Auch dieser Abschnitt ist mit HdG unterzeichnet. Bei der Lektüre verspüren wir diesmal nichts mehr von Fouquets Gedankenwelt, vielmehr handelt es sich um eine Zusammenfassung von Hallers Schrift «De partibus sensilibus et irritabilibus corporis humani» von 1752. Es handelt sich vor allem um die Auseinandersetzung, die Haller in den vorangehenden Jahren mit denjenigen Autoren hatte, welche die Sehnen empfindlich nannten. (NB Hallers Entdeckung der Unempfindlichkeit bzw. der NichtInnervation der Sehnen erwies sich als falsch.)

Der erste Satz in Hallers Artikel «sensibilité» heißt diesmal:

«La sensibilité fait le caractère essentiel de l'animal. Ce qui sent, est un animal, ce qui ne sent pas, ne l'est point... Sentir à l'égard de l'homme, c'est appercevoir dans l'âme un changement à l'occasion de l'impression que les corps qui nous environnent font sur les nerfs... L'organe du sentiment c'est le nerf. Tout ce qui blesse le nerf... cause un sentiment que nous appelons douleur... On ne doute point de la sensibilité du nerf.» ${ }^{20}$

Mit dem Ausdruck «caractère essentiel de l'animal» verspüren wir noch einen schwachen Anklang an den Vitalismus. Im übrigen werden wir an Lockes «sensation» erinnert, die nun ganz eindeutig in den Nerv lokalisiert wird. Zwar wurde diese Lokalisation schon vor Haller propagiert, aber nicht mit derselben Emphase und an einem Ort wie der Enzyklopädie, die eine weite Verbreitung erfuhr. Es erstaunt denn auch nicht, wenn William Cullen die gesamte Pathologie auf einem kranken Nervensystem (1769) begründet sah und den Terminus Neurose für Krankheit einführte. Als Abart dieser Neurosenpathologie kann der Brownianismus gelten, der um 1800 zur Modeströmung wurde.

\subsubsection{Marie-François-Xavier Bichat}

Mit Hallers apodiktischen Aussagen war aber die Frage um die Sensibilität keineswegs stillgelegt. Bichat (1771-1803), obwohl sehr jung verstorben, war ein sehr einflußreicher Lehrer. In seiner «Anatomie descriptive» 
(1801-1803) ist nicht nur von der Nervensensibilität die Rede. Er führt dazu noch den Begriff der «sensibilité organique» hinzu. Darunter versteht er die Sensibilität der Pflanzen, die kein Nervensystem haben, und die Sensibilität des menschlichen sog. Parenchyms (Leber, Niere, Bindegewebe usw.). Es war für Bichat also nicht nur der Nerv sensibel, sondern impliziterweise der ganze menschliche Körper. Mit dem Begriff der «sensibilité organique» schleicht sich wiederum ein Stück Vitalismusdenken in die Doktrin der Sensibilität ein, der möglicherweise die Erkenntnis der Rolle des sympathisch/vegetativen Nervensystems vorwegnimmt.

\section{Rückblick auf das 18. und Ausblick auf das 19. Jahrhundert}

Bis zu einem gewissen Grade vermischte sich demnach die Diskussion über die Sensibilität mit derjenigen über die Vitalkraft. Bald wurden die beiden Begriffe auseinandergehalten, bald waren es nur zwei verschiedene Namen für ein und dasselbe Gemeinte. Während Vitalismus im großen ganzen eher ein Ausdruck der Naturkundigen war, wurde die Doktrin der Sensibilität der Sensibilismus - eher von Ärzten vertreten.

Der Sensibilismus geht, wie wir nachgezeigt haben, auf Lockes Begriff von der «sensation» zurück. Alle Autoren waren sich darüber einig, daß die geistig/seelischen Strukturen auf der Verarbeitung der Sinnesempfindung beruhen, so wie es heute noch von der Naturwissenschaft und der Medizin angenommen wird.

Auffallend ist, daß in der Nachfolge von Condillac und auch von Haller vor allem der Tastsinn und der Schmerz als «sensation» im Lockeschen Sinne aufgefaßt wurden. Gehör- und Gesichtssinn waren unabhängige Untersuchungsrichtungen und hatten mit «Sensibilität» wenig zu tun.

Die Diskussion um die Sensibilität ebbte im 19. Jahrhundert nur langsam ab. In «Dictionnaire des sciences médicales» (1812-1822) finden wir wiederum eine 32 Seiten starke Abhandlung über das Thema Sensibilität, die zwar viel Traditionelles enthält, aber durchaus den zeitgenössischen Rahmen berücksichtigt. Er ist von Piorry gezeichnet. Pierre Adolphe Piorry (1794-1879) war ein prominenter Arzt aus Poitiers, der dann am Hôtel Dieu in Paris wirkte. Wie selbstverständlich stützt er sich auf Lockes «sensation», die er «étincelle vitale» nennt. Piorry betont, daß es ein und derselbe Name für dieses eine Phänomen gebe: Fakultät, Kraft, Irritabilität, Exzitabilität, Tonus, Tonizität usw. Wir hören hier das Echo auf die Diskussion um den Vitalismus und den damals modischen Brownianismus. Piorry wird spezifi- 
scher, wenn er die Frage stellt, was denn nun eigentlich unter Sensibilität verstanden werden solle, und ob die Nerven die einzigen Träger der tierischen Sensibilität seien. Diese letztere Frage verneint er gleich zu Beginn, indem er auf Bichats «sensibilité organique» verweist. Ferner stellt er eine Stufenreihe der nervösen Sensibilität auf und findet z.B. die Sympathicusganglien weniger sensibel als die Nervenstränge. Es folgt ein Überblick auf die sensiblen Teile unter pathologischen Bedingungen. Schließlich schreibt er wiederum der Haut die größte Sensibilität zu. Tastsinn und Schmerz gehören auch bei Piorry noch zusammen. Er referiert über die Versuche Hallers und Voltas. Seine Definition der Sensibilität lautet:

«C'est la propriété inhérente aux corps organisés.» ${ }^{21}$

Im Anhang zu Piorrys Artikel folgt ein Literaturverzeichnis. Der erste Titel über Sensibilität datiert von 1753. Daraus ersehen wir, daß die Diskussion um die Sensibilität um die Mitte des 18. Jahrhunderts begann, was gut mit den Tatsachen übereinstimmt, die wir zuvor erwähnt haben.

Auch das Experiment wurde angewendet, um dem Phänomen Tastsinn/Schmerz näherzukommen. Seit 1830 unternahm bekanntlich Ernst Heinrich Weber (1795-1878) seine berühmten Versuche über die Sensibilität der Haut ${ }^{22}$, wobei er weit geringere als Schmerzreize (Hautverformung, Gewichtsunterschiede usw.) als Experimentsstimulus zur Anwendung brachte. Er unterscheidet zwischen einem sensiblen Tastsinn an sich und einem Temperatursinn als zwei Modalitäten. Das Webersche Gesetz über die Sensibilität bei Gewichtsunterschieden setzt den Tastsinn in eine mathematische Relation. Die Schmerzerregung spielt bei seinen Versuchen eine untergeordnete Rolle. Dennoch bleibt der Schmerz auch für ihn eine Funktion der Hautsinnesorgane, aber nicht des Tastsinnes ${ }^{23}$. Noch im Lexikon «Larousse médical illustré» (Aufl. 1912) wird das Webersche Ästhesiometer als gängiges Prüfungsinstrument des Tastsinnes beschrieben, was es bis heute geblieben ist.

Mit den Weberschen Versuchen wurde der Weg freigesetzt, daß Tastsinn und Schmerzempfindung zwei eigene Forschungsrichtungen werden konnten. Das führte dann schließlich im 20. Jahrhundert zur Identifizierung der sog. Nozizeptoren, den Strukturen im Nervensystem, die zwar immer, wie geahnt, in der Haut liegen, aber vom Tastsinn getrennt sind.

Was mit Lockes «sensation» 1690 begann, wurde im 18. Jahrhundert zu einer spezifischen Art des Sensibilismus, indem es vor allem der Tastsinn und 
der Schmerz waren, die mit der «sensation» identifiziert wurden. Es entstand gewissermaßen eine Ideologie der Sensibilität. Erst Mitte des 19. Jahrhunderts wurden Getast und Schmerz gesondert untersucht, so daß heute Sensibilität, wie eingangs erwähnt, ein neurologischer, mit Kautelen auch ein physiologischer Begriff geworden ist.

\section{Anmerkungen}

1 Diese und alle folgenden biographischen Einzelheiten sind entnommen aus: Kenneth Dewhurst, John Locke, Physician and Philosopher, A Medical Biography, London 1963.

2 Robert G. Frank Jr., Harvey and the Oxford Physiologists, Berkeley/Los Angeles/London 1980.

3 Kenneth Dewhurst, Willis' Oxford Lectures, Oxford 1980.

4 loc. cit. sub no. 1.

5 David E. Wolfe, Sydenham and Locke on the limits of anatomy, in: Bull. Hist. Med. 35, 1961, 193-220.

6 Patrick Romanell, Locke and Sydenham, in: Bull. Hist. Med. 32, 1958, 293-321.

7 Alfred W. Gubser, Charles Barbeyrac und Sydenham, Diss. med. Zürich 1964.

7a Kenneth Dewhurst, Locke's Essay on Respiration, in: Bull. Hist. med. 34, 1960, 257-273.

8 Alfred Meyer und Raymond Hierons, On Thomas Willis' Concepts of Neurophysiology, in: Med. Hist. 9, 1965, 1-16 und 142-169; Hans-Rudolf Isler, Thomas Willis, Stuttgart 1965.

9 Antoinette Stettler, Geschichtliches über den Schmerz in Pathologie, Nervenphysiologie und Mentalität, in: Gesnerus 42, 1985, 433-454.

10 loc. cit. sub. no. 3, S. $65 / 66$.

11 John Locke, An Essay Concerning Human Understanding, Book II chap. 1, sect. 2.

12 ibid.

13 Etienne Bonnot de Condillac, Traité des Sensations, Paris 1754, ed.: Georges Lyon, Paris 1921, S. 49.

14 Lorin Anderson, Charles Bonnet and the Order of the Known, Dordrecht/Boston/London 1982, Kap. 4.

15 Albrecht von Haller, Empfindliche Teile des Körpers, ed.: Karl Sudhoff, Leipzig 1922, S. 14 .

16 Erna Lesky, Albrecht von Haller und Anton de Haen im Streit um die Lehre der Sensibilität, in: Gesnerus 16, 1959, 16-46.

17 Bernhard Josef Gottlieb, Bedeutung und Auswirkungen des Hallischen Professors und königlich/preußischen Leibarztes Georg Ernst Stahl auf den Vitalismus des 18. Jahrhunderts, insbesondere auf die Schule von Montpellier, in: Nova Acta Leopoldina, 12, 89, Halle 1943, 425-502.

18 Encyclopédie des sciences, arts et métiers..., Erstausgabe, Stichwort «sensibilité», Bd.15, S. 38 .

19 Ed. Karl Rothschuh, Von Boerhave zu Berger, Stuttgart 1964, S. 14 ff.

20 loc. cit. sub no. 18, Supplementband 4, 1777, Stichwort «sensibilité», S. 776. 
21 Dictionnaire des sciences médicales, Paris 1812-22, Stichwort «sensibilité».

22 Ernst Heinrich Weber, Der Tastsinn und das Allgemeingefühl, 1846.

23 Ursula Bueck-Rich, Ernst Heinrich Weber und der Anfang der Physiologie der Hautsinne, Diss. med. Zürich 1970.

\section{Weitere Literatur zum Thema}

Hans Buess, Physiologenstreit vor 200 Jahren, in: Experientia 2/4, 1946, 1-4.

Ernst Cassirer, Die Philosophie der Aufklärung, Tübingen 1932.

Friedrich Hoffmann, Fundamenta medicinae (1695), ed.: Lester S. King, London/New York 1971 (englische Übersetzung).

Richard Koch, War Georg Ernst Stahl ein selbständiger Denker?, in: Arch. Gesch. Med. (Sudhoff), 18, 1926, 20-51.

Lester S. King, Stahl and Hoffmann, A Study in 18th Century Animism, in: Jour. Hist. Med., 19, 1964, 118-130.

L.-J. Rather, G. E. Stahl's psychological Physiology, in: Bull. Hist. Med. 35, 1961, 37-49.

K. E. Rothschuh, Zur Geschichte der physiologischen Reizmethodik, in: Gesnerus 23, 1966, 147-159.

G. Rudolph, Diderots «Eléments de physiologie», in: Gesnerus 24, 1967, 24-45.

G. Sauder, Empfindsamkeit, Stuttgart 1974.

Richard N.Schwab, The History of Medicine in Diderots Encyclopédie, in: Bull. Hist. Med., $32,1958,216-223$.

Owsei Temkin, The Classical Roots of Glisson's Doctrine of Irritation, in: Bull. Hist. Med., 38, 1964, 297-328.

\section{Summary}

The paper opens with a description of John Lockes as a medical man. It then pursues the influence of his concept of the sensation (Essay Concerning Human Understanding, 1690) upon the French philosophical and medical establishment, especially the French Encyclopedists, where the concept of sensibility is formed. The influence of vitalism on sensibility is discussed and a short survey of the development in the 19th century is given.

Dr. med. Antoinette Stettler

Kirchenfeldstraße 45

CH-3005 Bern 